\title{
CONVEXITY FOR A SIMPLY CONNECTED $p$-ADIC GROUP
}

\author{
BY ALLAN J. SILBERGER
}

Communicated by Barbara Osofsky, April 24, 1975

In [4] Kostant showed that the set of Iwasawa double cosets which intersect a given Cartan double coset in a semisimple Lie group corresponds to a certain convex subset in the Lie algebra of a maximal torus of the group. As a consequence, he established that representatives for the double cosets relative to a maximal compact subgroup of a semisimple Lie group may be chosen in the unipotent radical of a minimal parabolic subgroup. We announce here analogues of these results for a simply-connected $p$-adic group.

I wish to thank Roger Howe and Jacques Tits for valuable conversations during the time I was thinking about this problem.

Let $\mathbf{G}$ be a connected simply-connected semisimple algebraic group defined over a $p$-adic field $\Omega$. Let $G$ denote the group of $\Omega$-rational points of $\mathbf{G}$. Then $G$ is a locally compact totally disconnected group. Borel and Tits [1] and Bruhat and Tits [2] have shown that $G$ has a structure theory which is similar in many ways to that of a semisimple Lie group.

Let $\mathbf{P}$ be a minimal parabolic $\Omega$-subgroup of $\mathbf{G}$. Then $\mathbf{P}$ is a split product $\mathbf{P}$ $\mathbf{P}=\mathbf{M N}$ in which $\mathbf{M}$ is connected and reductive and $\mathbf{N}$ is the unipotent radical of $\mathbf{P}$. Let $\mathbf{A}$ be the maximal $\Omega$-split torus in the center of $\mathbf{M}$. For simplicity, we denote the group of $\Omega$-points of each of the above algebraic groups by the corresponding ordinary capital letter.

Let ${ }^{\circ} A$ be the maximal compact subgroup of $A$. Then $A /{ }^{\circ} A$ is a free $\mathrm{Z}$ module of rank equal to the $\Omega$-rank of $\mathbf{G}$. We call $a=\left(A /{ }^{\circ} A\right) \otimes_{\mathrm{Z}} \mathbf{R}$ the Lie algebra of $A$ and write $H: A \rightarrow \mathfrak{a}$ for the natural map which imbeds $A /^{\circ} A$ as a lattice in $\boldsymbol{a}$. To any rational character $\chi$ of $\mathbf{A}$ we associate a linear functional on a by setting $\log |\chi(a)|=\langle\chi, H(a)\rangle(a \in A)$. The relative Weyl group $W=$ $N_{G}(A) / Z_{G}(A)$ operates on $A$ and $\mathfrak{a}$. There is a root system in the dual $\mathfrak{a}^{*}$ of $\mathfrak{a}$ associated to the restriction to $\mathbf{A}$ of the adjoint representation of $\mathbf{G}$. Choosing a $\boldsymbol{W}$-invariant scalar product on $\boldsymbol{a}$, we regard this root system as a subset of $\boldsymbol{a}$. Let $N$ correspond to a set of positive roots and let $\bar{N}$ be the $\Omega$-points of the unipotent radical of the opposite parabolic subgroup, corresponding to the negative roots. Write $\mathfrak{a}^{+}$[respectively, $+\mathfrak{a}$ ] to denote the (closed) positive chamber in $\mathfrak{a}$ [respectively, the cone consisting of nonnegative linear combinations of the positive roots]. The mapping $H$ extends to $M$; denoting the kernel of $H$ in $M$ as ${ }^{\circ} M$, we see that $H$ also imbeds $M /{ }^{\circ} M$ as a lattice in $\mathfrak{a}$. Let $M^{+}=H^{-1}\left(\mathfrak{a}^{+}\right)$and $+M=$ $H^{-1}(+\mathbf{a})$.

We choose a particularly "good" maximal compact subgroup $K$ of $G$ (i.e.

AMS (MOS) subject classifications (1970). Primary $20 \mathrm{G} 25$. 
corresponding to the origin in an apartment of the building of $G$ and such that $K \supset B \supset P \cap K=P \cap B, B$ a certain Iwahori subgroup of $G$ ), the existence of which is guaranteed by Bruhat's and Tits' theory, and recall that $G$ has, with respect to $K$, both a Cartan decomposition $G=K M^{+} K$ and an Iwasawa decomposition $G=N M K$. We have the bijections $K \backslash G / K \leftrightarrow M^{+} /{ }^{\circ} M$ and $N \backslash G / K \leftrightarrow$ $M /{ }^{\circ} M$.

For $S \subset \mathcal{U}$, we write $C(S)$ to denote the convex closure of $S$ and $W \cdot S$ to denote the Weyl group orbit of $S$. Our main result (cf. Theorem 4.1 of [4]) is

ThEOREM. Let $m, m^{\prime} \in M$. Then the double cosets $K m K$ and $\mathrm{Nm}^{\prime} K$ intersect if and only if $H\left(m^{\prime}\right) \in C(W \cdot H(m))$.

Since $0=H(1) \in C(W \cdot x)$ for all $x \in \mathfrak{a}$, we have

Corollary. $G=K N K$.

The following simple geometric lemma translates "only if" into a result [2, 4.4.4(iii)] of Bruhat-Tits.

Lemma 1. Let $x \in \mathfrak{a}^{+}$and set $\mathfrak{a}(x)=\left\{y \in \mathfrak{a}^{+} \mid x-y \in+\mathfrak{a}\right\}$. Then $W \cdot \mathbf{a}(x)=C(W \cdot x)$.

It follows by inspection of the defining integrals that the zonal spherical functions on $G$, evaluated at fixed $m \in M$ and regarded as functions on a (or rather on $\mathfrak{a}^{*}$ ), may be interpreted as generating functions for the measures of the intersections $K m K \cap N m^{\prime} K \quad\left(m^{\prime} \in M\right)$. The Weyl group invariance of such a function on $a^{*}[5]$ or, what is the same, the equivalence of all principal series representations of $G$ in a Weyl group orbit $[3, \S 8]$ implies

Lemma 2. Let $m, m^{\prime} \in M$. Then $K m K$ intersects $N^{\prime} m^{\prime} K$ for $N=N^{\prime}$ if and only if the same is true for some (i.e. for every) $N^{\prime}$ conjugate to $N$ under $W$.

The "if" part of our theorem is an easy consequence of the following structural result.

LemmA 3. Let $B$ and $\bar{N}$ be as above. Then $B \bar{N} K=B+M K$.

Details and proofs will appear elsewhere.

\section{REFERENCES}

1. A. Borel and J. Tits, Groupes réductifs, Inst. Hautes Études Sci. Publ. Math. No. 27 (1965), 55-150. MR 34 \#7527.

2. F. Bruhat and J. Tits, Groupes réductifs sur un corps local, Inst. Hautes Études Sci. Publ. Math. No. 41 (1972), 5-251. MR 48 \#6265.

3. Harish-Chandra, Harmonic analysis on reductive p-adic groups, Proc. Sympos. Pure Math., vol. 26, Amer. Math. Soc., Providence, R.I., 1974, pp. 167-192.

4. B. Kostant, On convexity, the Weyl group, and the Iwasawa decomposition, Ann. Sci. École Norm. Sup. (1974). 
5. I. G. Macdonald, Spherical functions on a group of p-adic type, Publ. Ramanujan Inst., no. 2, Ramanujan Institute, University of Madras, Madras 5, India, 1971.

SONDERFORSCHUNGSBEREICH, “THEORETISCHE MATHEMATIK”, UNIVERSITÄT BONN, BONN, FEDERAL REPUBLIC OF GERMANY

DEPARTMENT OF MATHEMATICS, UNIVERSITY OF MASSACHUSETTS, AMHERST, MASSACHUSETTS 01002

Current address: Department of Mathematics, Cleveland State University, Cleveland, Ohio 44115 